OPEN ACCESS

Edited by:

Kun Zhou,

Nanyang Technological University,

Singapore

Reviewed by:

Haibao LU,

Harbin Institute of Technology, China

Xinli Xiao,

Harbin Institute of Technology, China

*Correspondence:

Zhengyi Song

zysong@jlu.edu.cn

Specialty section:

This article was submitted to

Smart Materials,

a section of the journal

Frontiers in Materials

Received: 18 January 2021

Accepted: 12 April 2021

Published: 07 May 2021

Citation:

Ren L, Li B, Liu Q, Ren L, Song Z,

Zhou $X$ and Gao P (2021) 4D Printing

Dual Stimuli-Responsive Bilayer

Structure Toward Multiple

Shape-Shifting.

Front. Mater. 8:655160.

doi: 10.3389/fmats.2021.655160

\section{D Printing Dual Stimuli-Responsive Bilayer Structure Toward Multiple Shape-Shifting}

\author{
Luquan Ren ${ }^{1,2}$, Bingqian Li1, Qingping Liu' ${ }^{1,2}$, Lei Ren ${ }^{1,2}$, Zhengyi Song ${ }^{1,2 *}$, Xueli Zhou ${ }^{1,2}$ \\ and Peng Gao ${ }^{1}$
}

${ }^{1}$ Key Laboratory of Bionic Engineering (Ministry of Education), Jilin University, Changchun, China, ${ }^{2}$ Weihai Institute of Bionics, Jilin University, Weihai, China

4D printing has been attracting widespread attention because its shape and performance can change under stimuli. The existing 4D printing technology is mostly limited to responsive to single stimulus, which means that the printing structure can only change under a pre-specified stimulus. Here we propose a 4D printing strategy with dual stimuli-responsive shape-shifting that responds to both temperature and water, by using a direct ink writing 3D printing method to deposit a polyurethane elastomer material with water-swelling characteristics on a heat-shrinkage shape memory polymer material to form a bilayer structure. Based on the systematic study of the adapted printing parameters of the polyurethane elastomer, the effect of programmable variables on the deformation shape was investigated. The diversified printing structure exhibits rich structural changes under one or both of the two stimuli of temperature and water. This research provides a universal multiple stimuli-responsive 4D printing method, which can effectively improve the intelligent responsiveness of $4 \mathrm{D}$ printing structures by combining multiple smart materials.

Keywords: 4D printing, multi-stimuli-response, bilayer structures, multiple shape-shifting, transformable soft gripper

\section{INTRODUCTION}

$4 \mathrm{D}$ printing refers to that the shape, property or functionality of 3D printed objects can change over time in response to external stimuli (Kuang et al., 2019; Lui et al., 2019; Rastogi and Kandasubramanian, 2019; Shafranek et al., 2019), such as water, heat, light, electricity, etc. 4D printing has broad application prospects in many fields such as aerospace (Akbari et al., 2018), drug delivery (Ursan et al., 2013), biomedical equipment (Gao et al., 2016; Yang et al., 2019), and flexible electronic devices (Zarek et al., 2016), and has been attracting widespread attention. To date, most of the research on $4 \mathrm{D}$ printing involves the shape-shifting of $3 \mathrm{D}$ printed materials or structures. The shape-changing mechanism of $4 \mathrm{D}$ printing can be summarized as either using smart materials, such as hydrogels (Champeau et al., 2020; Hu et al., 2020), shape memory polymers (SMP) (GonzálezHenríquez et al., 2019; Zhang et al., 2019c,d; Kong et al., 2020), liquid crystal elastomers (LCE) (Kotikian et al., 2018; Zhang et al., 2019a), etc., or creating mismatch strain within the printed object during the printing process by the combination of different responsive materials (Tibbits, 2014) or the variation of printing process parameters (van Manen et al., 2017), and then the deformation is generated in response to stimuli after printing. 
In the research field of $4 \mathrm{D}$ printing, multiple stimuli-response is of great significance for broadening the practical application of the printed structure in different environments (Karis et al., 2017; Han et al., 2018). By adding functional fillers to a certain type of smart material, the material has more responsiveness than the original one, which is one of the common strategies for achieving multi-response $4 \mathrm{D}$ printing. This draws on the existing multiresponse composite method (Hu et al., 2018; Li et al., 2018). In such strategies, thermal is generally used as a basic stimulation. Researchers added carbon nanotubes, ferroferric oxide or other fillers into the SMPs, LCEs or thermally responsive hydrogels to enable the material with the capability to convert electricity to heat (Han et al., 2018; Liu et al., 2019), magnetism to heat (Lin et al., 2019; Zhang et al., 2019b), or light to heat (Cui et al., 2019; del Barrio and Sánchez-Somolinos, 2019), thereby realizing multi-responsive $4 \mathrm{D}$ printing. The composites formed from a thermally responsive material and a material responsive to $\mathrm{pH}$ (Hu et al., 2020; Lee et al., 2020) or cations (Kirillova et al., 2017; Duigou et al., 2019) also have multiply stimuli responsiveness. In addition, the water-responsive material can also be used as the basis material, and then the response of another material can change the moisture (Duigou et al., 2019), thereby controlling the change of the structure. In essence, these stimuli-responses are ultimately attributed to one stimulation. When multiple stimuli are applied, the resulting responses are consistent. This is not conducive to the use of a single printing structure in multiple environments.

Another pathway to achieve multiple stimuli-response $4 \mathrm{D}$ printing is to construct a bilayer structure by combining two materials with different response properties. Compared with the above-mentioned methods, the two layer materials is independent of each other in response to environmental stimuli, and can produce more shapes during the deformation process, thus enriching the deformation mode of the single structure. Regrettably, there are extremely few studies on multiply stimulusresponse $4 \mathrm{D}$ printing at this stage.

By referring to the bilayer materials each with different responsiveness constructed by other methods (Zhang et al., 2016; Boothby and Ware, 2017; Xiao et al., 2018; Wang et al., 2018), it can be found that the structure has more responsiveness, which greatly enriches the pattern of structural changes. Using the respectively intelligent properties of the two material to stimulate deformation, the structure can deform as pre-defined by programming the type of environmental stimuli. For example, Boothby et al. combined a thermally responsive LCE with a waterresponsive polymer to form a dual stimuli-responsive shapechanging bilayer structure (Kirillova et al., 2017). Yang et al. (2019) present a facile method to prepare dual-responsive bilayer hydrogels, consisting of a thermo-responsive layer and a saltresponsive layer through in situ sequential radical polymerization (Duigou et al., 2019). However, the structural styles formed by these methods are still relatively simple. $4 \mathrm{D}$ printing method can significantly increase the complexity of the structural design, resulting in more shape-changing.

In this article, we propose a $4 \mathrm{D}$ printing strategy based on the water-swellable polyurethane (PU) elastomer and the heatshrinkage shape memory polymer polyvinyl chloride (SMPVC) bilayer structure that can produce programmable deformation in response to temperature and water. We investigated the rheological properties of $\mathrm{PU}$, showing good printability. Then the effect of the process parameters of direct inks writing $3 \mathrm{D}$ printing on the printing filament width was studied. Further studies on the parameters that may affect the deformation behavior of the PU-SMPVC bilayer structure, including PU thickness, width and angle, were carried out. Based on the research results, we designed and manufactured a variety of shape-shifting structures with diversified morphology changes. Finally, three shapes deformed by the same printing structure under different stimuli were used as three soft gripper to verify the gripping capabilities.

\section{RESULTS}

\section{PU-SMPVC Bilayer Structures}

This article proposes a $4 \mathrm{D}$ printing strategy that can achieve programmable shape changes in response to both temperature and water. As depicted in Figure 1, by means of direct ink writing 3D printing method, the water-swellable PU elastomer is deposited on the thermal-shrinkable shape memory polymer to form a bilayer structure. After the PU is completely cured by absorbing moisture in the air, the PU/SMPVC bilayer structure was obtained by cutting along the outline of the printed sample for subsequent experiments. In response to different stimuli, the mismatch strain caused by wate-swelling or thermal-shrinkage drives the printed bilayer structure to produce various shapeshifting. By programming the stimuli, the shape-shifting of the PU-SMPVC bilayer structure can be effectively controlled.

\section{Investigation of Rheological Properties and Printability}

In order to realize the printing of PU-SMPVC bilayer structures, the printability of water-swellable PU has been investigated. First, the rheological properties of PU were measured. As shown in Figure 2A, the apparent viscosity of PU gradually decreases with the increase of the shear rate at room temperature of $25^{\circ} \mathrm{C}$, showing a shear thinning rheological behavior. Properly elevated temperature $\left(40^{\circ} \mathrm{C}\right)$ can decrease the viscosity of $\mathrm{PU}$, which is more conducive to smooth out material from extrusion head. Figure 2B shows the changing of the storage modulus $\left(\mathrm{G}^{\prime}\right)$ and loss modulus $\left(\mathrm{G}^{\prime \prime}\right)$ of the PU as function of shear strain at a constant frequency under different temperature conditions. With the gradual increase of the shear strain, $G^{\prime}$ to $G^{\prime \prime}$ gradually decrease. During the process, $G^{\prime}$ changes from greater than $G^{\prime \prime}$ to less than $G^{\prime \prime}$, indicating the transition of the material from solid to viscoelastic under high shear strain conditions, which is suitable for the material extrusion process. In addition, a proper increase in temperature $\left(40^{\circ} \mathrm{C}\right)$ will decrease the values of both $\mathrm{G}^{\prime}$ and $\mathrm{G}^{\prime \prime}$, but still keep them the similar change trends.

Since this experiment adopts pneumatic-based direct ink writing technology (DIW), the coordination of printing moving speed and air pressure determines the quality of the printed filaments. Figure 3A shows whether continuous lines can be extruded under different speeds and different air pressures at $25^{\circ} \mathrm{C}$. The line width change of extrudable filaments with 


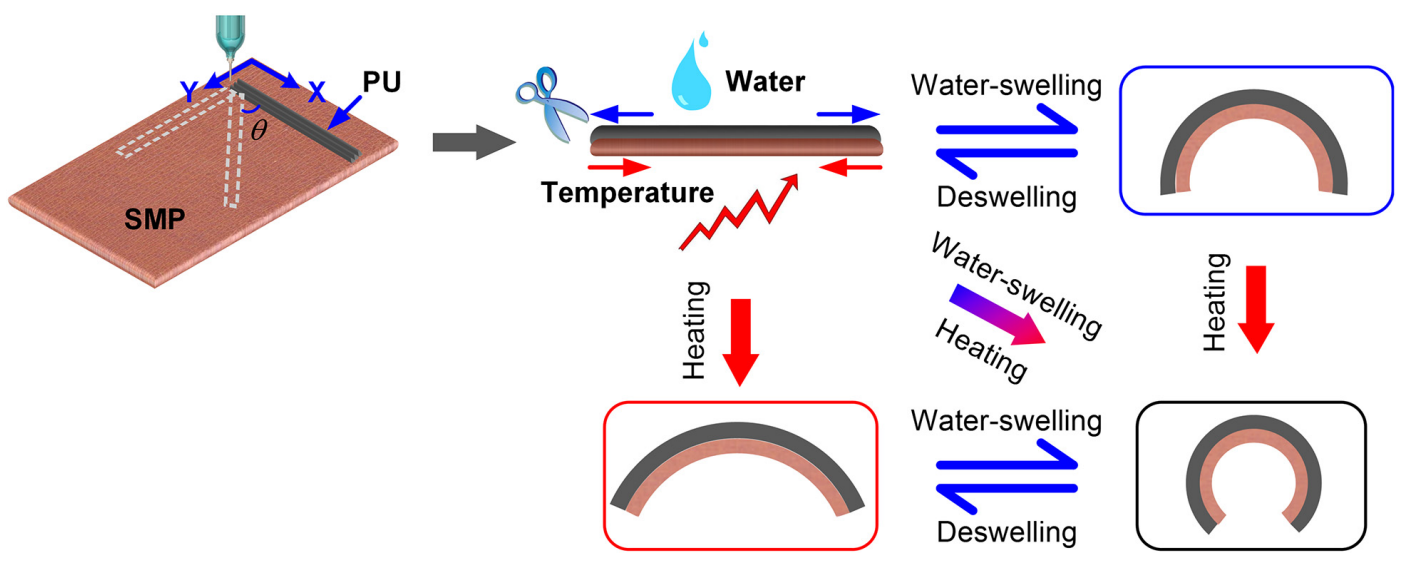

FIGURE 1 | Schematic diagram of manufacturing and shape-shifting under different stimuli of 4D printed PU-SMPVC bilayer structures.
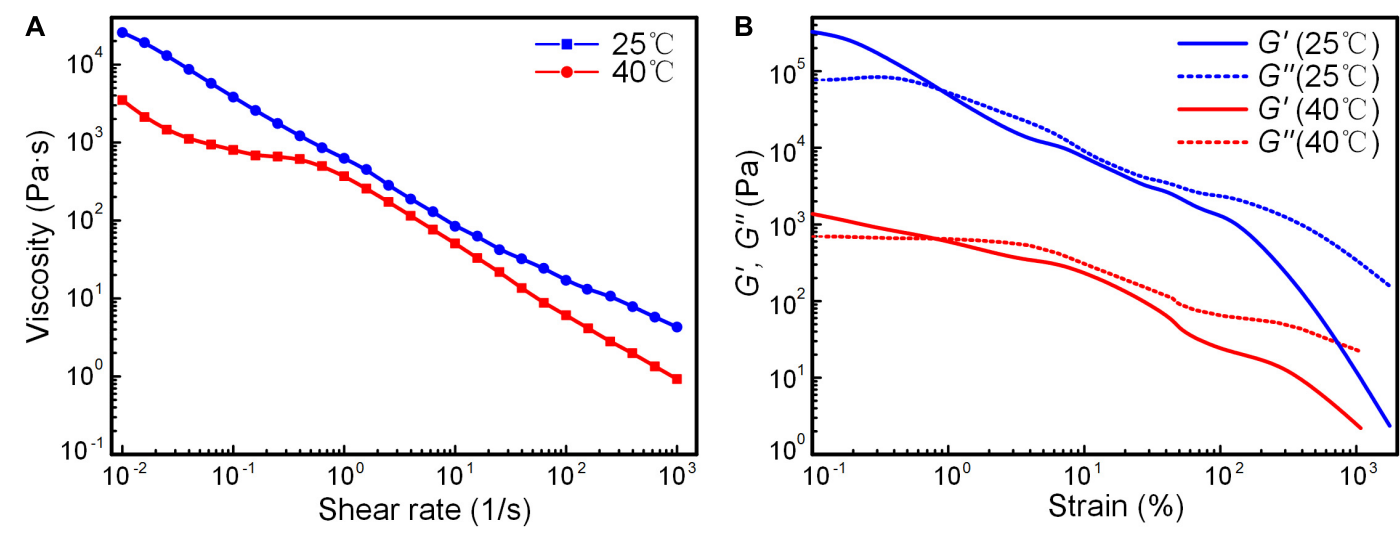

FIGURE 2 | Rheological properties of the PU elastomer under 25 and $40^{\circ} \mathrm{C}$. (A) Apparent viscosity as functions of shear rates for inks with different temperature. (B) Storage and loss moduli as functions of shear strain with different temperature.

different speeds and air pressures is shown in Figure $3 \mathbf{B}$, from which we can conclude that the greater the air pressure and the slower the speed, the greater the width of the extruded filament, Under the condition of air pressure of $0.6 \mathrm{MPa}$ and speed of $5 \mathrm{~mm} / \mathrm{s}$, the maximum line width can reach about $0.75 \mathrm{~mm}$, which is basically twice the diameter of the extrusion head $(0.41 \mathrm{~mm})$. According to the obtained data, the distribution colormap of filament width and air pressure/speed are drawn out (Figure 3C), and on this basis, a appropriate speed and air pressure can be selected to achieve quantitative filament width printing. Figures 3D-F depicts whether the filament can be extruded smoothly and the change of the filament width as different speeds and pressures at $40^{\circ} \mathrm{C}$. Compared with the condition of $25^{\circ} \mathrm{C}$, under the same speed and air pressure, the width of the extruded filament at $40^{\circ} \mathrm{C}$ is wider. The maximum line width exceeds $1 \mathrm{~mm}$, indicating that the extruded material volume is more. This also reflects the improvement of the rheological properties of the ink, which is consistent with the conclusion obtained from the rheological test. In the case of the same air pressure, in order to achieve the same filament width, the required printing speed is a bit faster at $40^{\circ} \mathrm{C}$ than that at $25^{\circ} \mathrm{C}$, which can improve the printing efficiency to a certain extent. Therefore, the printing temperature used in this experiment is set as $40^{\circ} \mathrm{C}$.

Figures 4A-C shows the examples of $4 \mathrm{D}$ printed $\mathrm{PU}$ elastomer under a combination of parameters with a printing temperature of $40^{\circ} \mathrm{C}$, a speed of $12 \mathrm{~mm} / \mathrm{s}$, and an air pressure of $0.4 \mathrm{MPa}$ (Figure 3F). These examples are raster structure, ring column and butterfly shape, respectively. All three printed samples can swell by absorbing water with a linear swelling rate of approximately 1.5. From the weight change of the three structures shown in Table 1 before and after water-swelling, the weight of the ring column and butterfly- shaped structure after water-swelling is 2.9 times the initial weight, while the raster structure is increased to 3.66 times. This may be due to the more water contact area in the structure, which can be capable to absorb water more fully.

The swollen samples can deswell at room temperature or under heating and return to their original shapes. Under the condition of $60^{\circ} \mathrm{C}$, it can be seen from the weight changes of different samples (Figure 4D) that the sample with raster 

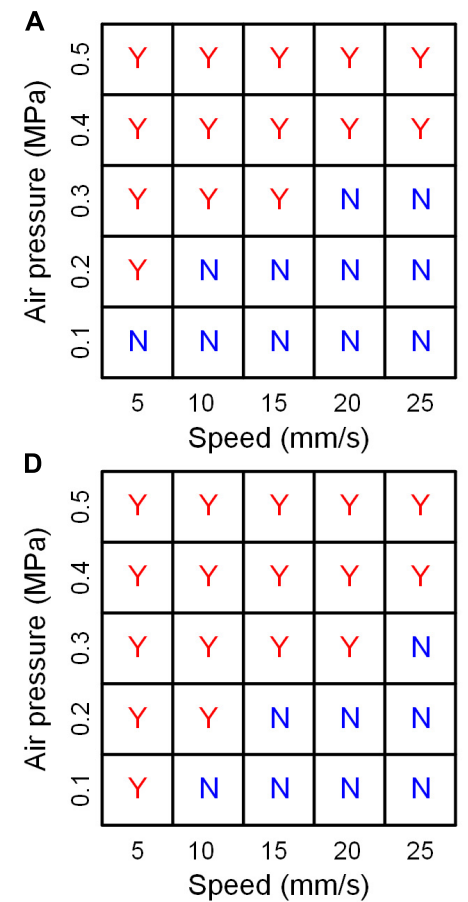
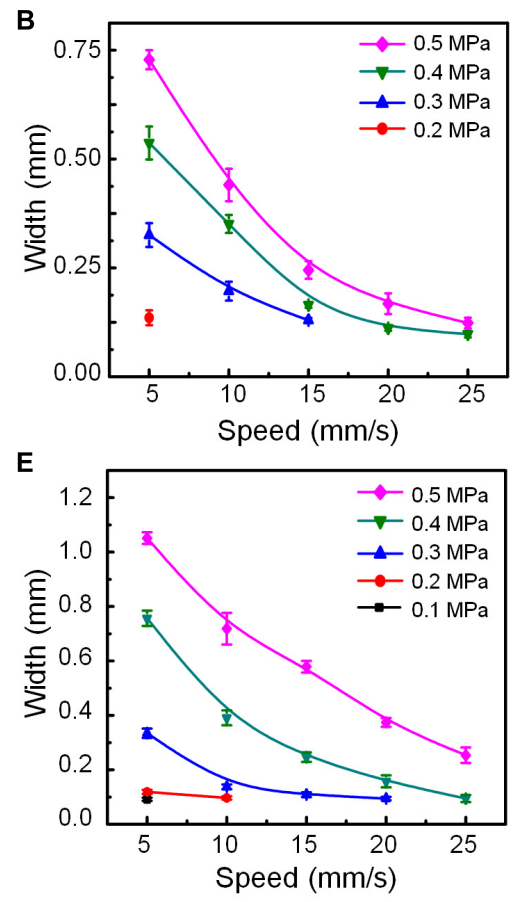
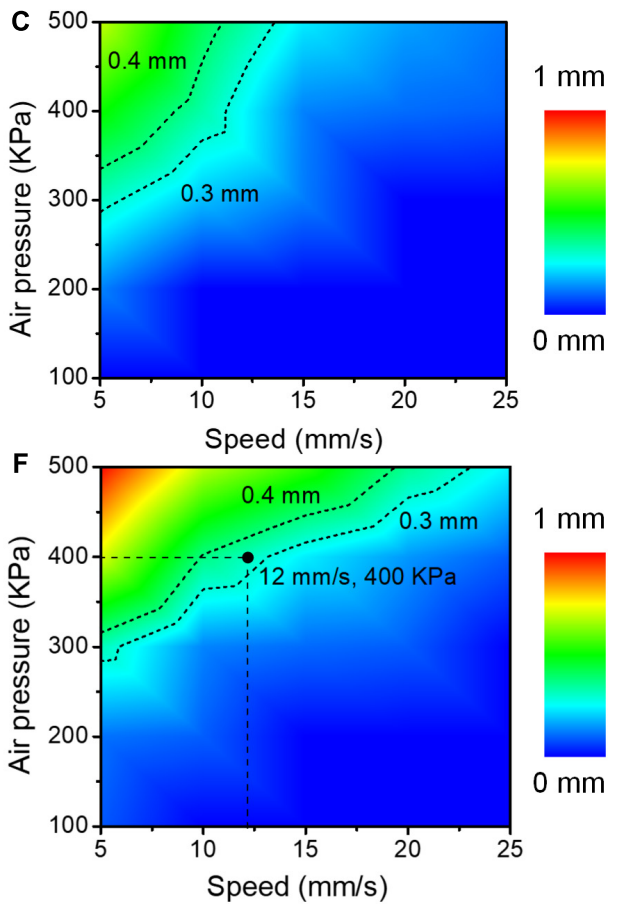

FIGURE 3 | Investigation of the effect of air pressure and moving speed on the printed filaments at $25^{\circ} \mathrm{C}(\mathbf{A}-\mathbf{C})$ and $40^{\circ} \mathrm{C}(\mathbf{D}-\mathbf{F})$.

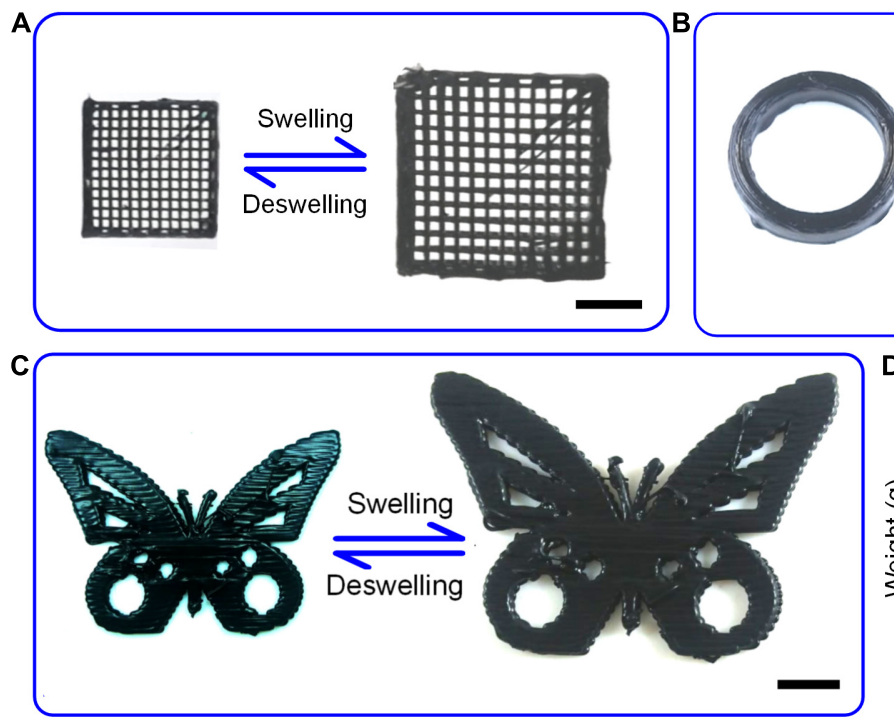

$\frac{\text { Swelling }}{\sum_{\text {Deswelling }}}$
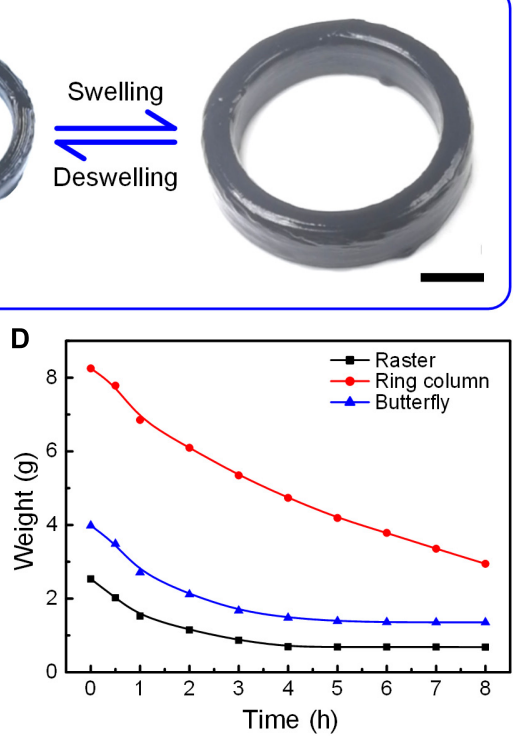

FIGURE 4 | Three printed PU samples with raster structure (A), ring column (B), and butterfly shape (C) and their shapes after water-swelling. (D) Changes in the weight of three printed samples during the deswelling process at room temperature.

structure can recover to the original shape at the fastest speed ( $4 \mathrm{~h})$, while the butterfly shaped sample can recover the initial shape in about $5 \mathrm{~h}$. However, because there are more areas that are not in direct contact with the air, the rate of water loss of the sample with ring column shape is the slowest, requiring more than $8 \mathrm{~h}$. If the swollen samples are placed at room temperature, it will take longer to fully recover the original shapes.

\section{Relationship Between Printing} Parameters and Deformation

The deformability of the printed bilayer structure in response to temperature or/and water was investigated. Figure $\mathbf{5 A}$ shows the relationship between the deformability of the bilayer structures printed in the radial direction and the thickness of the printed PU layer. With the gradual increase of the printing thickness, 
TABLE 1 | Weight change of the printed structures before and after absorbing water.

\begin{tabular}{lccc}
\hline $\begin{array}{l}\text { Shape of the } \\
\text { printed samples }\end{array}$ & $\begin{array}{c}\text { Initial } \\
\text { weight (g) }\end{array}$ & $\begin{array}{c}\text { Temporary } \\
\text { weight (g) }\end{array}$ & $\begin{array}{c}\text { Temporary/ } \\
\text { Initial }\end{array}$ \\
\hline Raster structure & 0.708 & 2.532 & 3.66 \\
Ring column & 2.846 & 8.251 & 2.90 \\
Butterfly & 1.367 & 3.984 & 2.91 \\
\hline
\end{tabular}

the degree of bending deformation caused by the shrinkage of the SMPVC layer gradually decreases (Figure 5B), which is caused by the increasing shape-morphing inhibition of the PU layer thickness. Similarly, the bending deformation caused by moisture is also affected by the thickness. The greater the thickness, the larger the bending radius (Figure $5 \mathrm{C}$ ). In the case of integrating two stimuli (Figure 5D), both two work together to increase the bending effect. The final bending radius will increase with the increase in thickness. In addition, the relationship between the deformability of the bilayer structures printed in the radial direction and the width of the printed PU layer was also investigated ( $c f$. Supplementary Figure S1). The results demonstrate that the width of PU elastomer has little effect on the temperature/water response of the bilayer structure.

Then, the relationship between the deformation mode of the bilayer structure printed at an oblique angle of $45^{\circ}$ to the radial direction and the thickness of the printed PU layer was investigated. Herein, the bending deformation responding to water is no longer considered because its deformation is consistent with the bending deformation caused by water along the radial direction. Figure $\mathbf{6 A}$ shows the shapes of the printed samples with different PU layer thickness after thermal stimulus and theirs shape transition after water-swelling. With the gradual increase of the printing thickness, the degree of spiral formed by thermal shrinkage gradually decreases, which is manifested as the gradual increase in pitch, and the spiral radius also shows a gradually increasing trend (Figures 6B,C). When the thermalinduced spiral shape samples are placed in a water environment, the spiral degree will change to a certain extent. The spiral pitch becomes smaller, but the radius becomes slightly larger. On the whole, the pitch and degree still keep increasing as the thickness increases. Moreover, the width has almost no effect on the spiral deformation of the bilayer structure printed at an oblique angle of $45^{\circ}$, which means that the pitch and radius before and after deformation are independent of the width ( $c f$. Supplementary Figure S2).

In view of the difference between the radial shrinkage and axial shrinkage of the SMPVC layer, the angle of the printed PU layer will also affect the deformation mode of the PU-SMPVC bilayer structure. As shown in Figure 7, when responding to temperature $\left(85^{\circ} \mathrm{C}\right)$, as the angle gradually increases, the printed structure produces regular deformation, which is manifested from pure bending deformation to spiral deformation. The pitch increases with the angle, but the radius remains unchanged.

\section{Multi-Morphic Deformation}

After learning the influence of printing parameters on structural deformation, a variety of samples with multi-morphic structural deformation were designed and manufactured. Based on the effect of the printing thickness of the PU layer on the shape changing of the bilayer structure, a structure with a gradient in thickness was printed, as shown in Figure 8. When the structure respond to water alone or temperature alone, it can evolve into an involute spiral shape.

Considering that the angle can affect the deformation of the bilayer structure under temperature, we printed a bilayer structure with a quadrant shape, which can realize the transformation of the inclination angle from $0^{\circ}$ to $90^{\circ}$, as shown in Figure 9. This structure can produce different morphological transformation under different stimuli. Utilizing the characteristics of anisotropic shrinkage of SMPVC can achieve the gradient change of pitch from 0 to infinity, showing a gradient spiral shape. Moreover, the level of stimulated temperature can affect the shrinkage degree of the SMPVC, thereby resulting in different deformation modes of the bilayer
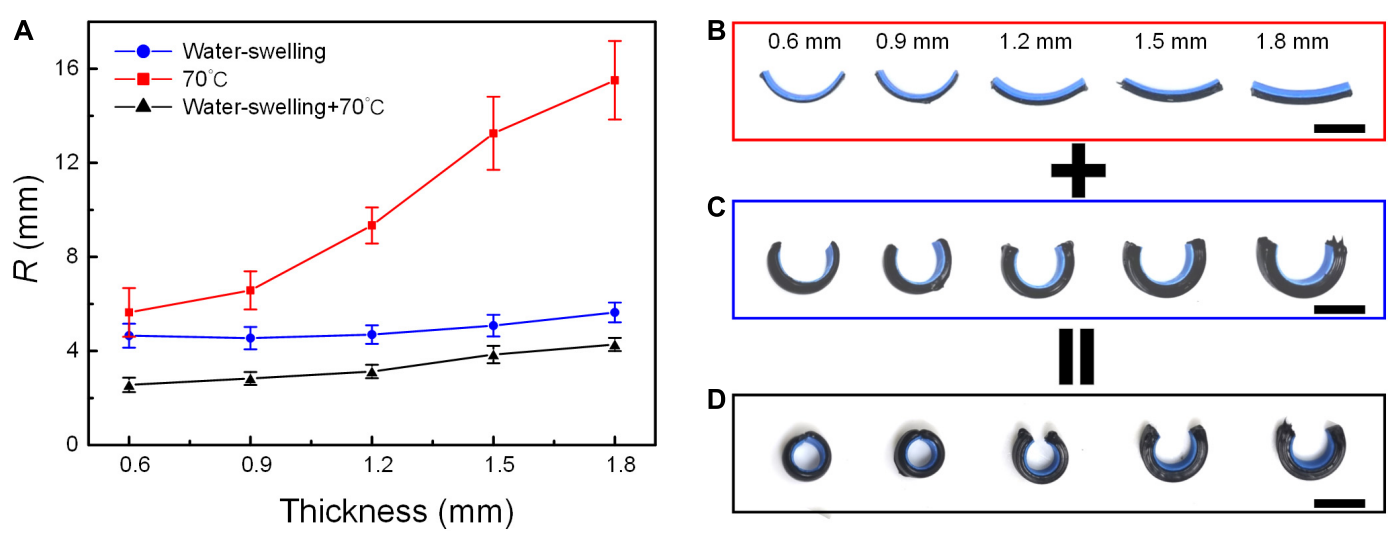

FIGURE 5 | The effect of the PU layer thickness printed in the radial direction on bending deformation of the PU-SMPVC bilayer structures. (A) Bending radius as a function of PU layer thickness in $70^{\circ} \mathrm{C}$, room temperature water and $70^{\circ} \mathrm{C}$ water. Images of bending deformation of the PU-SMPVC bilayer structures in $70^{\circ} \mathrm{C}(\mathbf{B})$, room temperature water (C) and $70^{\circ} \mathrm{C}$ water (D). 

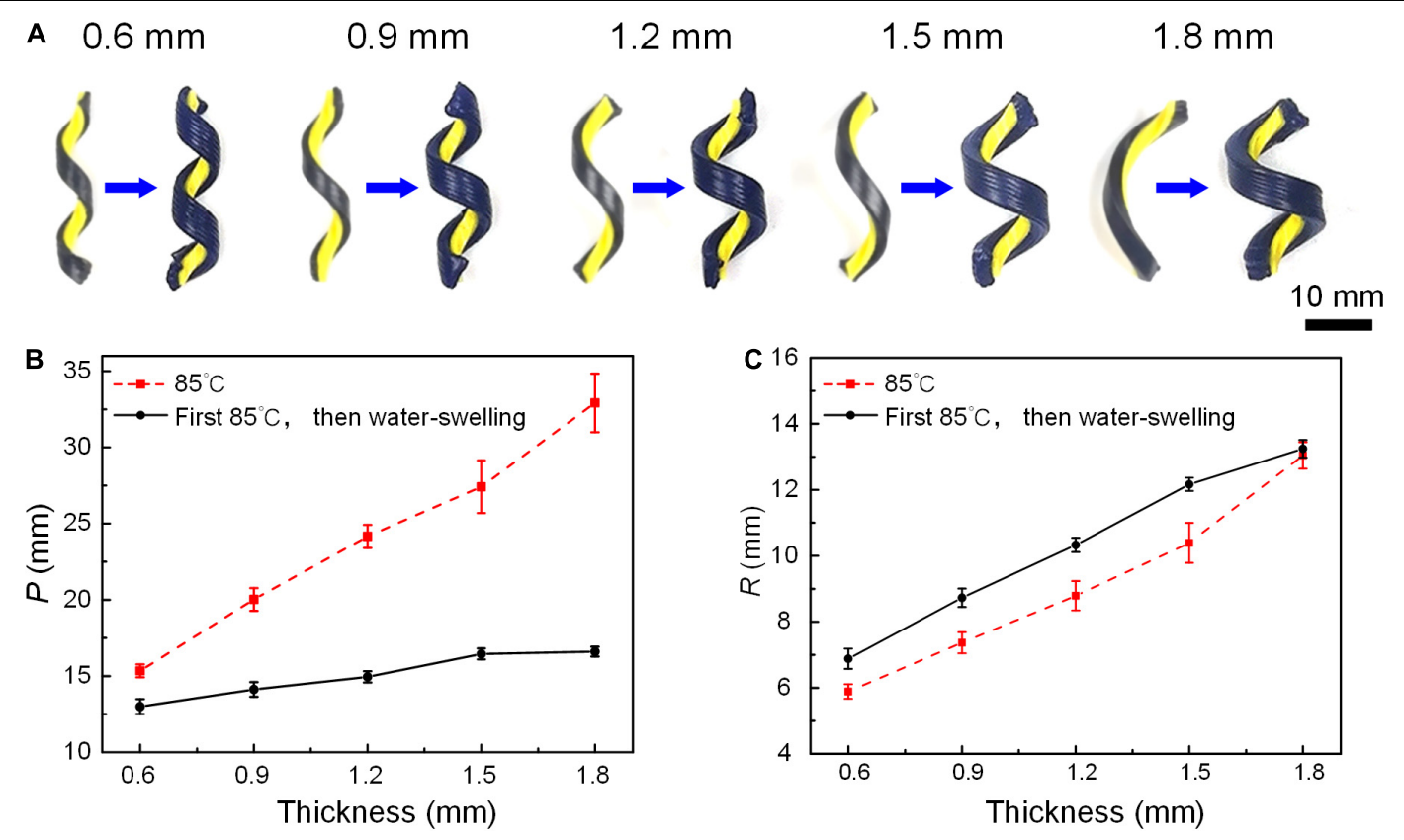

FIGURE 6 | The effect of the PU layer thickness printed at an oblique angle of $45^{\circ}$ on spiral deformation of the PU-SMPVC bilayer structures. (A) Images of spiral deformation of the PU-SMPVC bilayer structures generated in $85^{\circ} \mathrm{C}$ and the shifted shapes induced by water-swelling after thermally induced deformation. The values of pitch $\mathbf{( B )}$ and radius $(\mathbf{C})$ of thermally induced spiral deformation and under the combined action of water and heat $\left(85^{\circ} \mathrm{C}\right)$.

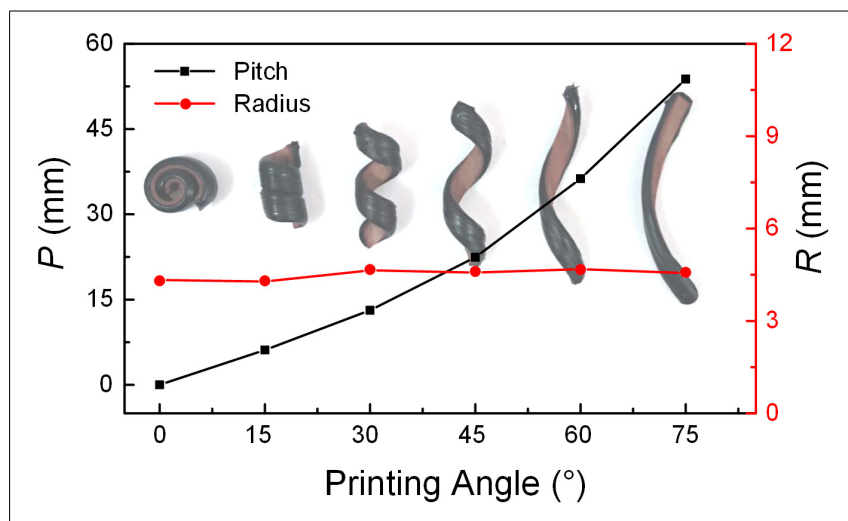

FIGURE 7 | Changes in the pitch and radius of the deformed PU-SMPVC bilayer structure in response to temperature $\left(85^{\circ} \mathrm{C}\right)$. The insets represent corresponding deformed shapes.

structure. As shown in the Figure, the gradient spiral shapes produced at 70 and $90^{\circ} \mathrm{C}$ are significantly different. The nonuniform spiral at high temperature $\left(90^{\circ} \mathrm{C}\right)$ is more obvious, and the spiral shape formed after water-swelling is also more prominent. In addition, by first undergoing water-swelling to deform into a circle, and then receiving thermal stimulus at different temperatures, different non-uniform spiral shapes can also be obtained.

Figure 10 shows the shape morphing of a bilayer structure with a printed semicircular shape under different stimuli, which is symmetrical along the axial direction. Both sides can produce gradient spiral deformation with opposite spiral directions in response to temperature. After water-swelling, the bending deformation effect enables the two sides to further spirally deform toward respective direction, forming a greater nonuniform spiral shape. Another way to get the final shape is to first achieve uniform bending deformation by water-swelling, and then receive thermal stimulation to obtain a bilaterally symmetrical non-uniform spiral structure.

Another example shown in Figure $\mathbf{1 1}$ is a bilayer structure composed of four branches with a quarter circle shape. When excited by water, the four branches will bend evenly, which finally makes the structure present a sequential overlapping pattern. In response to temperature, the four branches will produce nonuniform spiral deformation. The two opposite branches have the same deformation pattern. Based on this, the four branches can be divided into two groups, one of which is spirally deformed from the inside to the outside, and the other is the opposite. When responding to two stimuli, the synergy of the waterinduced bending effect and the thermally induced non-uniform spiral effect creates a new form. More structures with multiple deformations are shown in the Supplementary Material ( $c f$. Supplementary Figures S3, S4).

\section{Transformable Soft Gripper}

Harnessing the multiple shape-shifting of the PU-SMPVC bilayer structure, a transformable soft gripper is designed, as shown in Figure 12A. The PU layer is distributed on the SMPVC layer in a crisscross shape with an angle of $45^{\circ}$ in the radial direction of the SMPVC layer. In response to water, the four branches bend evenly in the SMPVC direction to form a curved soft gripper. When responding to temperature, the four branches are uniformly 


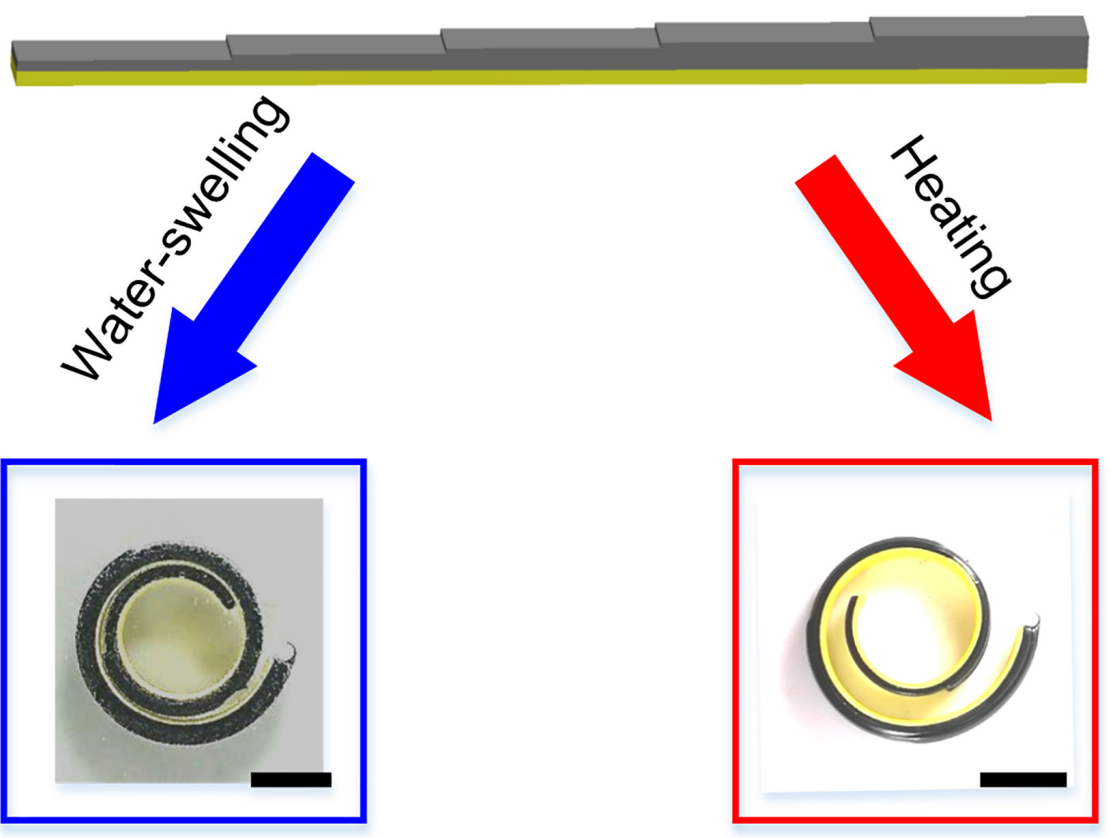

FIGURE 8 | PU-SMPVC bilayer structure with a gradient PU layer thickness, which can produce an involute shape undergoing water-swelling or heating.

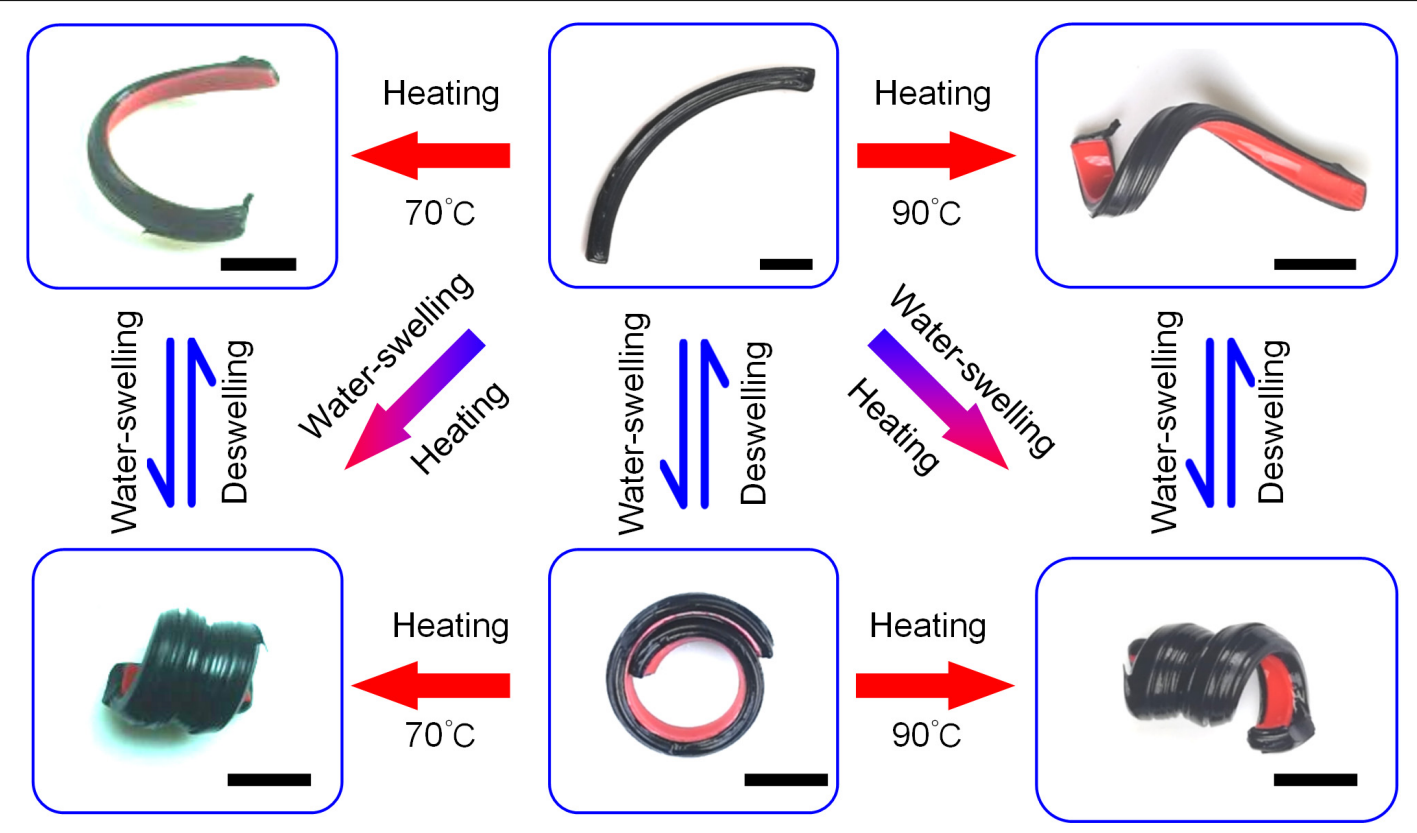

FIGURE 9 | Deformation of a PU-SMPVC bilayer structure with a quadrant shape in response to different stimuli.

spiral in the SMPVC direction. According to the spiral direction, the structure can be divided into two groups with opposite spiral directions. The two adjacent gripping arms intersect at one point, forming a spiral soft gripper. In response to the dual stimuli of temperature and water, the water-induced bending effect and the thermally induced spiral effect cause the structure to produce a greater degree of spiral deformation toward the SMPVC side, resulting in the third deformation pattern. All the three 3D shapes transformed from the PU-SMPVC bilayer flat structure can be used as a soft gripper. The gripping capabilities of the three soft grippers are compared through the relationship between the output force and displacement during the process of gripping the fixed bolt, as shown in Figure 12B. It can be seen that the deformation caused by the dual stimuli produces the largest 

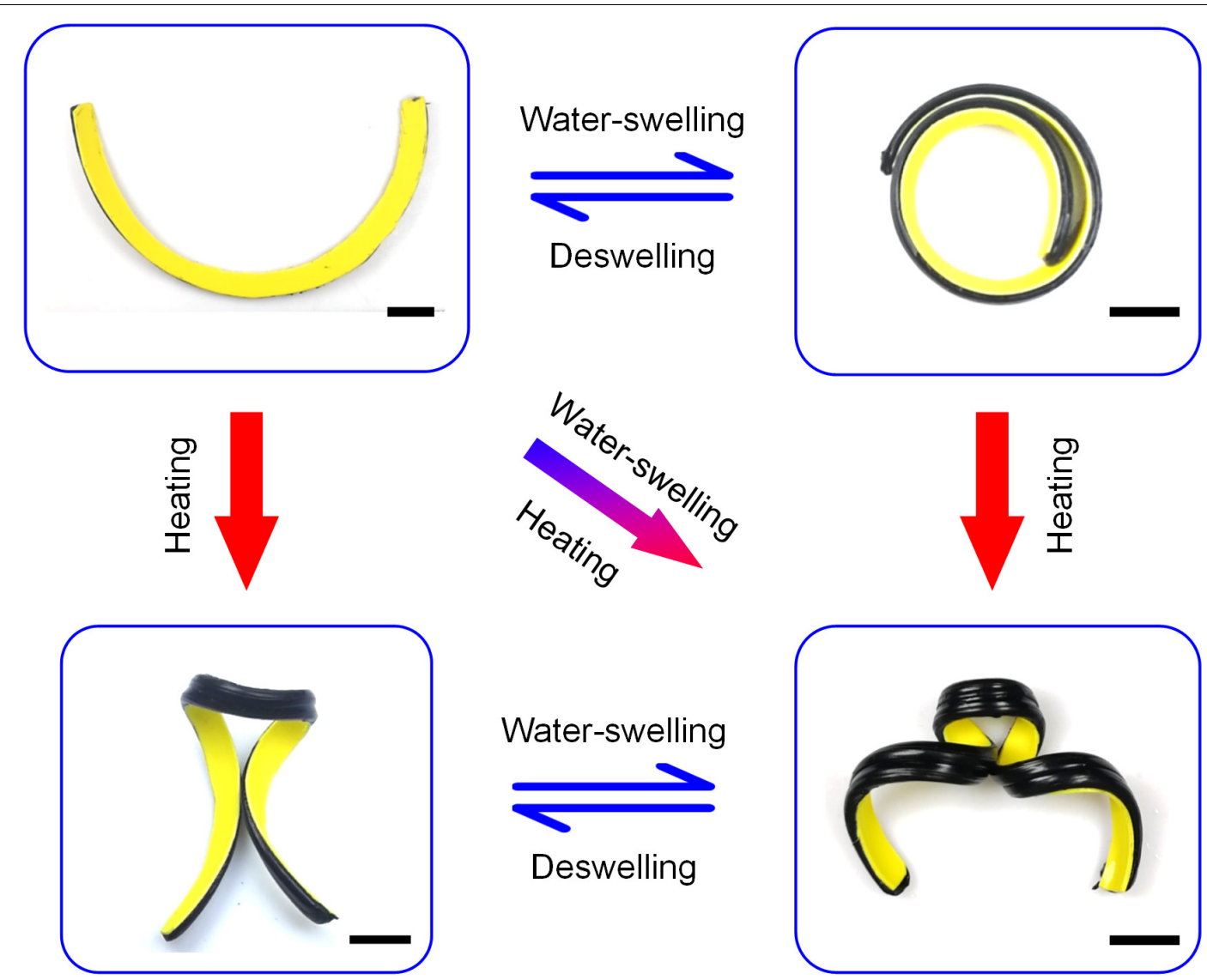

FIGURE 10 | Deformation of a PU-SMPVC bilayer structure with a semicircular shape in response to different stimuli.

force during the desorption process, while the desorption force generated only in response to water is the smallest, and the desorption force generated in response to temperature $\left(90^{\circ} \mathrm{C}\right)$ is in the middle. The result proves that the soft gripper under the dual stimuli has better gripping ability.

\section{CONCLUSION}

In this article, we demonstrated a facile and feasible 4D printing method that can achieve dual stimuli-responsive shape-shifting in response to temperature or/and water. Harnessing DIW 3D printing method to deposit the water-swelling $\mathrm{PU}$ material on the heat-shrinkage SMPVC material, a bilayer structure was construed. Moreover, the printability of PU enables the manufacture of complex deformation structures. In addition, the effects of printing thickness and printing angle on deformation ensures the shape-shifting diversity of the printing structure. Based on previous research, multiple multi-morphic deformation structures have been designed. Finally, the usefulness of dual stimuli-response is verified by a transformable soft gripper. This article presents a facile, universal multi-response 4D printing method, which can facilitate the intelligent responsiveness of $4 \mathrm{D}$ printed structures by combining multiple smart materials.

\section{MATERIALS AND METHODS}

\section{Materials}

The water-swellable PU elastomer material with a linear swelling ratio of $150 \%$ (Hengchuang Construction Engineering Material Co., Ltd., China) are used in this article. The material is in the form of a black gel and can be directly loaded into the syringe for subsequent printing. The glass transition temperature of $\mathrm{PU}$ is $-58.79^{\circ} \mathrm{C}$ ( $c f$. Supplementary Figure S5A). The thermal decomposition temperature of $\mathrm{PU}$ exceeds $200^{\circ} \mathrm{C}$ ( $c f$. Supplementary Figure S5B).

A commercial available heat-shrinkable PVC (Polyvinyl chloride, Volsun Co., Ltd., nominal thickness: $0.6 \mathrm{~mm}$ ) with a 2:1 nominal shrinking ratio in the radial direction and a negligible axial shrinkage ratio was used. In addition, according to the previous research literature, the degree of this SMPVC shrinkage is related with the temperature (Janbaz et al., 2016). The higher the temperature, the greater the shrinkage.

\section{Characterization}

A rotational viscometer (Discovery HR-30, America) equipped with $20 \mathrm{~mm}$ diameter parallel was employed for investigating the rheological properties of the PU paste. The apparent viscosity was determined in a flow sweep mode at shear rates of $0.01-10 \mathrm{~s}^{-1}$. 

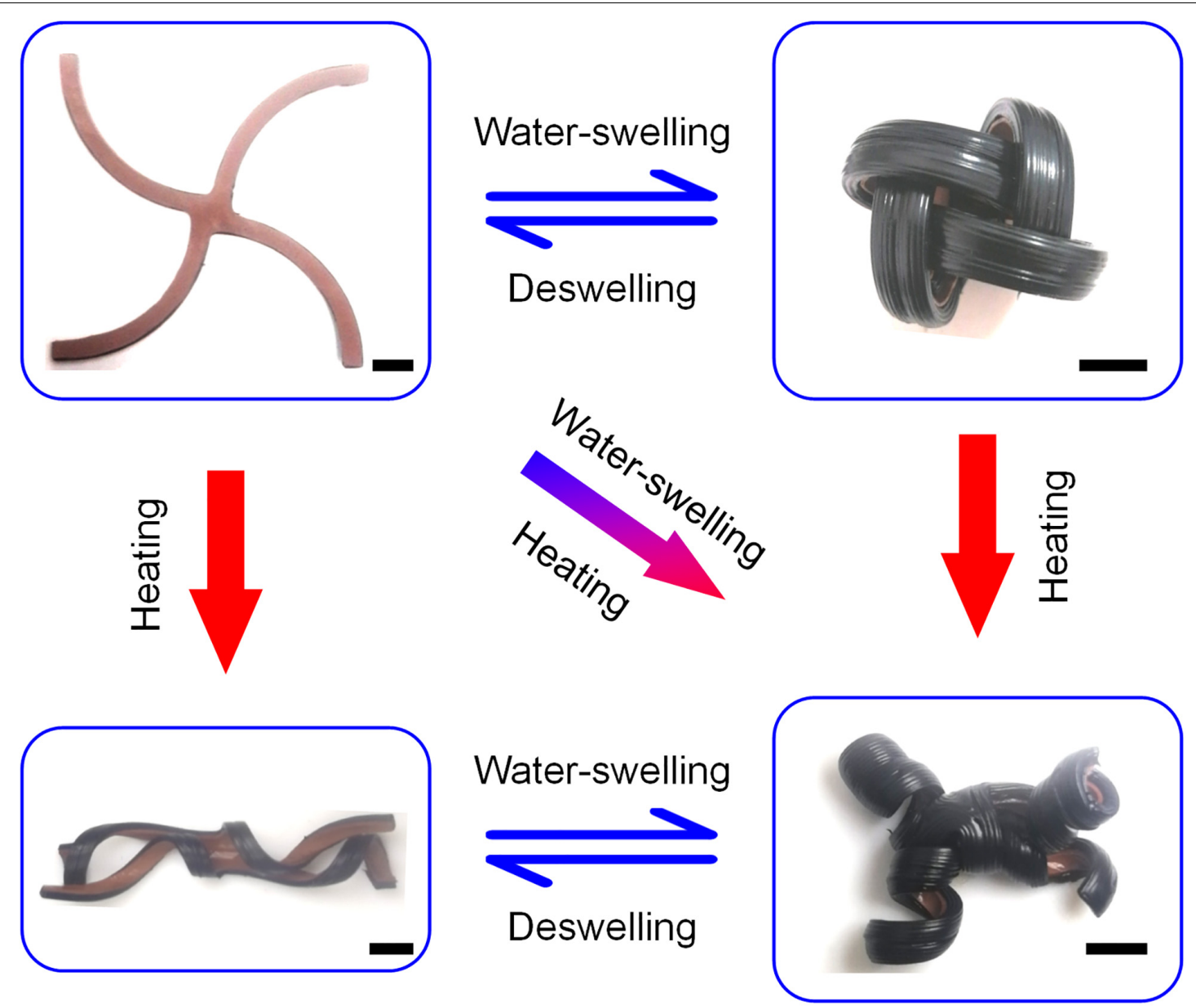

FIGURE 11 | Deformation of a PU-SMPVC bilayer structure composed of four branches with a quarter circle shape in response to different stimuli. Each branch is obtained by rotating the adjacent branch clockwise.
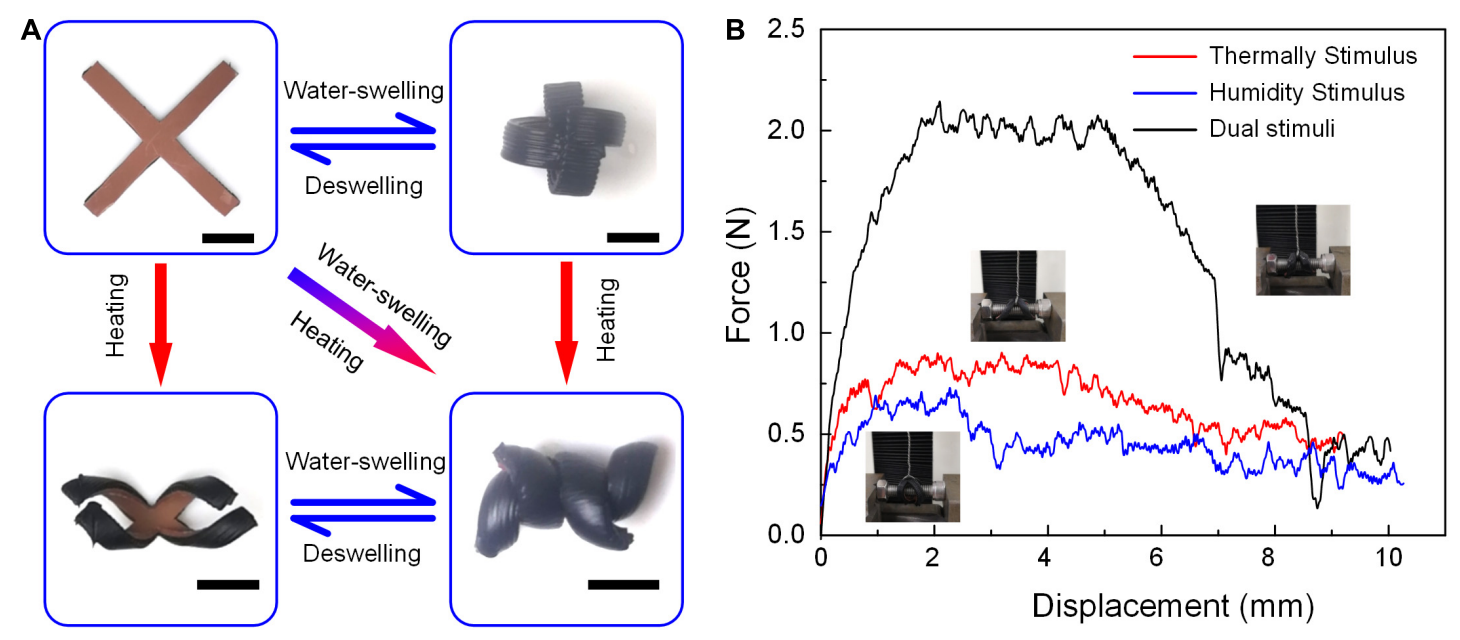

FIGURE 12 | Transformable soft gripper. (A) Deformation of a PU-SMPVC bilayer structure in a crisscross shape with an angle of $45^{\circ}$ in the radial direction of the SMPVC layer in response to different stimuli. (B) The relationship between the desorption force and displacement generated by three soft grippers with different shapes in the process of gripping a fixed bolt. 
Gripping tests were carried out using a universal testing machine (ZQ-990A, China). A bolt with a diameter of $6 \mathrm{~mm}$ is fixed by a vise clamp. The soft grippers with different shapes formed under different stimuli are attached on the bolt in the form of grasping. The force generated during the upward motion $(5 \mathrm{~mm} / \mathrm{min}$ ) was recorded to evaluate gripping ability of different soft grippers.

The values of the filament width, bending radius, helical pitch and helical radius in this article were all measured from the captured image using image analysis software (Digimizer, MedCalc Software, Belgium).

\section{D Printing and Shape-Shifting}

All printed objects were manufactured by using our selfdeveloped pneumatic assisted direct inks writing (DIW) 3D printer. In the printing process, we used stainless steel needles (22G) with a inner diameter of $0.41 \mathrm{~mm}$ to print all objects. All prints in this study were conducted at a fixed layer height of $0.3 \mathrm{~mm}$.

During printing of the PU/SMPVC bilayer structure, a flat sheet of SMPVC polished with sandpaper (600-mesh) is fixed on the substrate. The PU elastomer filaments are deposited on the SMPVC layer through the extrusion head. After PU elastomer is thoroughly solidified by absorbing moisture in air (exceed 3 days), the PU/SMPVC bilayer structure was acquired by cutting along the outline of the printed PU filaments.

The morphed shapes in response to temperature, water, and both two stimuli shown in Figures 4-12 were obtained by submerging the specimens in the hot water bath with different temperature for 25-30 s, in the room temperature water bath for $3 \mathrm{~h}$, and first in the hot water bath with different temperature for 25-30 s, then in room temperature water bath for $3 \mathrm{~h}$, respectively.

\section{REFERENCES}

Akbari, S., Sakhaei, A. H., Kowsari, K., Serjouei, A., Zhang, Y. F., and Ge, Q. (2018). Enhanced multimaterial $4 \mathrm{D}$ printing with active hinges. Smart Mater. Struct. 27:065027. doi: 10.1088/1361-665X/aabe63

Boothby, J. M., and Ware, T. H. (2017). Dual-responsive, shape-switching bilayers enabled by liquid crystal elastomers. Soft Matter. 13, 4349-4356. doi: 10.1039/ c7sm00541e

Champeau, M., Heinze, D. A., Viana, T. N., de Souza, E. R., Chinellato, A. C., and Titotto, S. (2020). 4D printing of hydrogels: a review. Adv. Funct. Mater. 30:1910606. doi: 10.1002/adfm.201910606

Cui, H., Miao, S., Esworthy, T., Lee, S. J., Zhou, X., Hann, S. Y., et al. (2019). A novel near-infrared light responsive 4D printed nanoarchitecture with dynamically and remotely controllable transformation. Nano Res. 12, 1381-1388. doi: 10. 1007/s12274-019-2340-9

del Barrio, J., and Sánchez-Somolinos, C. (2019). Light to shape the future: from photolithography to 4D printing. Adv. Opt. Mater. 7:1900598. doi: 10.1002/ adom. 201900598

Duigou, A. L., Chabaud, G., Scarpa, F., and Castro, M. (2019). Bioinspired electrothermo-hygro reversible shape-changing materials by $4 \mathrm{D}$ printing. Adv. Funct. Mater. 29:1903280. doi: 10.1002/adfm.201903280

Gao, B., Yang, Q., Zhao, X., Jin, G., Ma, Y., and Xu, F. (2016). 4D bioprinting for biomedical applications. Trends Biotechnol. 34, 746-756. doi: 10.1016/j.tibtech. 2016.03.004

\section{DATA AVAILABILITY STATEMENT}

The original contributions presented in the study are included in the article/Supplementary Material, further inquiries can be directed to the corresponding author/s.

\section{AUTHOR CONTRIBUTIONS}

LR, QL, ZS, and LR participated in design and procedure planning of the experiment. BL, ZS, XZ, and PG were responsible for different parts of the experiment. All authors have contributed to the article, read, and approved the manuscript.

\section{FUNDING}

This work was supported by the Key Scientific and Technological Project of Jilin Province (20170204061GX), the National Key Research and Development Program of China (2018YFB1105100 and 2018YFF01012400), and the Scientific Research Project of Jilin Provincial Department of Education (JJKH20211117KJ).

\section{ACKNOWLEDGMENTS}

We would like to acknowledge PE Jiang for technical assistance of rheological testing.

\section{SUPPLEMENTARY MATERIAL}

The Supplementary Material for this article can be found online at: https://www.frontiersin.org/articles/10.3389/fmats. 2021.655160/full\#supplementary-material

González-Henríquez, C. M., Sarabia-Vallejos, M. A., and Rodriguez-Hernandez, J. (2019). Polymers for additive manufacturing and 4D-printing: materials, methodologies, and biomedical applications. Prog. Polym. Sci. 94, 57-116. doi: 10.1016/j.progpolymsci.2019.03.001

Han, D., Farino, C., Yang, C., Scott, T., Browe, D., Choi, W., et al. (2018). Soft robotic manipulation and locomotion with a $3 \mathrm{D}$ printed electroactive hydrogel. ACS Appl. Mater. Interfaces 10, 17512-17518. doi: 10.1021/acsami.8b0 4250

Hu, Y., Wang, Z., Jin, D., Zhang, C., Sun, R., Li, Z., et al. (2020). Botanical- inspired 4D printing of hydrogel at the microscale. Adv. Funct. Mater. 30:1907377. doi: 10.1002/adfm.201907377

Hu, Z., Zhang, D., Lu, F., Yuan, W., Xu, X., Zhang, Q., et al. (2018). Multistimuli-responsive intrinsic self-healing epoxy resin constructed by hostguest interactions. Macromolecules 51, 5294-5303. doi: 10.1021/acs.macromol. 8 b01124

Janbaz, S., Hedayati, R., and Zadpoor, A. A. (2016). Programming the shapeshifting of flat soft matter: from self-rolling/self-twisting materials to selffolding origami. Mater. Horiz. 3, 536-547. doi: 10.1039/C6MH00195E

Karis, D., Ono, R., Zhang, M., Vora, A., Storti, D., Ganter, M. A., et al. (2017). Cross-linkable multi-stimuli responsive hydrogel inks for direct-write $3 \mathrm{~d}$ printing. Polym. Chem. 8, 4199-4206. doi: 10.1039/c7py00831g

Kirillova, A., Maxson, R., Stoychev, G., Gomillion, C. T., and Ionov, L. (2017). 4D biofabrication using shape-morphing hydrogels. Adv. Mater. 29:1703443. doi: 10.1002/adma.201703443 
Kong, D., Li, J., Guo, A., and Xiao, X. (2020). High temperature electromagnetic shielding shape memory polymer composite. Chem. Eng. J. 408:127365. doi: 10.1016/j.cej.2020.127365

Kotikian, A., Truby, R. L., Boley, J. W., White, T. J., and Lewis, J. A. (2018). $3 \mathrm{D}$ printing of liquid crystal elastomeric actuators with spatially programed nematic order. Adv. Mater. 30:1706164. doi: 10.1002/adma.201706164

Kuang, X., Roach, D. J., Wu, J., Hamel, C. M., Ding, Z., Wang, T., et al. (2019). Advances in 4D printing: materials and applications. Adv. Funct. Mater. 29:1805290. doi: 10.1002/adfm.afdm201805290

Lee, Y. W., Ceylan, H., Yasa, I. C., Kilic, U., and Sitti, M. (2020). 3D-printed multi-stimuli-responsive mobile micromachines. ACS Appl. Mater. Interfaces 13, 12759-12766. doi: 10.1021/acsami.0c18221

Li, G., Hong, G., Dong, D., Song, W., and Zhang, X. (2018). Multiresponsive graphene-aerogel-directed phase-change smart fibers. Adv. Mater. 30:1801754. doi: 10.1002/adma.201801754

Lin, C., Lv, J., Li, Y., Zhang, F., Li, J., Liu, Y., et al. (2019). 4D-printed biodegradable and remotely controllable shape memory occlusion devices. Adv. Funct. Mater. 29:1906569. doi: 10.1002/adfm.201906569

Liu, Y., Zhang, F., Leng, J., Fu, K., Lu, X. L., Wang, L., et al. (2019). Remotely and sequentially controlled actuation of electroactivated carbon nanotube/shape memory polymer composites. Adv. Mater. Technol. 4:1900600. doi: 10.1002/ admt.201900600

Lui, Y. S., Sow, W. T., Tan, L. P., Wu, Y., Lai, Y., and Li, H. (2019). 4D printing and stimuli-responsive materials in biomedical aspects. Acta Biomater. 92, 19-36. doi: 10.1016/j.actbio.2019.05.005

Rastogi, P., and Kandasubramanian, B. (2019). Breakthrough in the printing tactics for stimuli-responsive materials: 4D printing. Chem. Eng. J. 366, 264-304. doi: 10.1016/j.cej.2019.02.085

Shafranek, R. T., Millik, S. C., Smith, P. T., Lee, C. U., Boydston, A. J., and Nelson, A. (2019). Stimuli-responsive materials in additive manufacturing. Prog. Polym. Sci. 93, 36-67. doi: 10.1016/j.progpolymsci.2019.03.002

Tibbits, S. (2014). 4D printing: multi-material shape change. Archit. Des. 84, 116-121. doi: 10.1002/ad.1710

Ursan, I. D., Chiu, L., and Pierce, A. (2013). Three-dimensional drug printing: a structured review. J. Am. Pharm. Assoc. 53, 136-144. doi: 10.1331/JAPhA.2013. 12217

van Manen, T., Janbaz, S., and Zadpoor, A. A. (2017). Programming 2D/3D shapeshifting with hobbyist 3D printers. Mater. Horiz. 4, 1064-1069. doi: 10.1039/ C7MH00269F
Wang, L., Jian, Y., Le, X., Lu, W., Ma, C., Zhang, J., et al. (2018). Actuating and memorizing bilayer hydrogels for a self-deformed shape memory function. Chem. Commun. 54, 1229-1232. doi: 10.1039/c7cc09456f

Xiao, S., Zhang, M., He, X., Huang, L., Zhang, Y., Ren, B., et al. (2018). Dual saltand thermoresponsive programmable bilayer hydrogel actuators with pseudointerpenetrating double-network structures. ACS Appl. Mater. Interfaces 12, 6351-6361. doi: 10.1021/acsami.9b17577

Yang, G. H., Yeo, M., Koo, Y. W., and Kim, G. H. (2019). 4D bioprinting: technological advances in biofabrication. Macromol. Biosci. 19:e1800441. doi: 10.1002/mabi.201800441

Zarek, M., Layani, M., Cooperstein, I., Sachyani, E., Cohn, D., and Magdassi, S. (2016). 3D printing of shape memory polymers for flexible electronic devices. Adv. Mater. 28, 4449-4454. doi: 10.1002/adma.201503132

Zhang, C., Lu, X., Fei, G., Wang, Z., Xia, H., and Zhao, Y. (2019a). 4D Printing of a liquid crystal elastomer with a controllable orientation gradient. ACS Appl. Mater. Interfaces 11, 44774-44782. doi: 10.1021/acsami.9b18037

Zhang, F., Wang, L., Zheng, Z., Liu, Y., and Leng, J. (2019b). Magnetic programming of 4D printed shape memory composite structures. Compos. Part A Appl. Sci. Manuf. 125:105571. doi: 10.1016/j.compositesa.2019.105571

Zhang, L., Desta, I., and Naumov, P. (2016). Synergistic action of thermoresponsive and hygroresponsive elements elicits rapid and directional response of a bilayer actuator. Chem. Commun. 52, 5920-5923. doi: 10.1039/c6cc01193d

Zhang, Y., Huang, L., Song, H., Ni, C., Wu, J., Zhao, Q., et al. (2019c). 4D printing of a digital shape memory polymer with tunable high performance. ACS Appl. Mater. Interfaces 11, 32408-32413.

Zhang, Y., Le, X., Jian, Y., Lu, W., and Chen, T. (2019d). 3D fluorescent hydrogel origami for multistage data security protection. Adv. Funct. Mater. 29:1905514. doi: 10.1002/adfm.201905514

Conflict of Interest: The authors declare that the research was conducted in the absence of any commercial or financial relationships that could be construed as a potential conflict of interest.

Copyright (C) 2021 Ren, Li, Liu, Ren, Song, Zhou and Gao. This is an open-access article distributed under the terms of the Creative Commons Attribution License (CC BY). The use, distribution or reproduction in other forums is permitted, provided the original author(s) and the copyright owner(s) are credited and that the original publication in this journal is cited, in accordance with accepted academic practice. No use, distribution or reproduction is permitted which does not comply with these terms. 